\title{
Molecular adaptation of a plant-bacterium outer membrane protease towards plague virulence factor Pla
}

\author{
Johanna Haiko ${ }^{1}$, Liisa Laakkonen ${ }^{1,2}$, Benita Westerlund-Wikström¹, Timo K Korhonen ${ }^{1 *}$
}

\begin{abstract}
Background: Omptins are a family of outer membrane proteases that have spread by horizontal gene transfer in Gram-negative bacteria that infect vertebrates or plants. Despite structural similarity, the molecular functions of omptins differ in a manner that reflects the life style of their host bacteria. To simulate the molecular adaptation of omptins, we applied site-specific mutagenesis to make Epo of the plant pathogenic Erwinia pyrifoliae exhibit virulence-associated functions of its close homolog, the plasminogen activator Pla of Yersinia pestis. We addressed three virulence-associated functions exhibited by Pla, i.e., proteolytic activation of plasminogen, proteolytic degradation of serine protease inhibitors, and invasion into human cells.

Results: Pla and Epo expressed in Escherichia coli are both functional endopeptidases and cleave human serine protease inhibitors, but Epo failed to activate plasminogen and to mediate invasion into a human endothelial-like cell line. Swapping of ten amino acid residues at two surface loops of Pla and Epo introduced plasminogen activation capacity in Epo and inactivated the function in Pla. We also compared the structure of Pla and the modeled structure of Epo to analyze the structural variations that could rationalize the different proteolytic activities. Epo-expressing bacteria managed to invade human cells only after all extramembranous residues that differ between Pla and Epo and the first transmembrane $\beta$-strand had been changed.

Conclusions: We describe molecular adaptation of a protease from an environmental setting towards a virulence factor detrimental for humans. Our results stress the evolvability of bacterial $\beta$-barrel surface structures and the environment as a source of progenitor virulence molecules of human pathogens.
\end{abstract}

\section{Background}

Analyses of genomic sequences of bacterial pathogens have given an unprecedented view into their biology and evolutionary processes $[1,2]$. A conclusion from these studies is that highly similar genes, many of which are associated with bacterial virulence, are found across great phylogenetic distances and in different genetic elements, which is indicative of horizontal gene transfer. These families of virulence factors - including toxins, transport systems, adhesins, and antibiotic resistance factors - have evolved by adaptive radiation of a functional progenitor molecule to and within other strains and species to support survival in differing ecological

\footnotetext{
* Correspondence: timo.korhonen@helsinki.fi

'Division of General Microbiology, Department of Biosciences, P.O. Box 56, FI 00014 University of Helsinki, Finland

Full list of author information is available at the end of the article
}

niches [2]. The adaptation, or "evolutionary fine-tuning" of virulence factors that results in increased fitness, can involve modification of catalytic efficiency or substrate specificity of an enzyme, or alteration of bacterial interactions with target cells [2]. The mechanisms of horizontal gene transfer and the functional diversity of bacterial toxin families and protein transport systems have been documented [2-4] but adaptive molecular evolution of bacterial virulence factors remains less understood in terms of altered structure/function relationships.

Yersinia pestis is the causative agent of plague, a zoonotic disease transmitted to humans usually by the bite of an infected flea [5]. The bacterium spreads from the intradermal infection site into lymph nodes, causing bubonic plague, and subsequently to blood and to lungs, leading to pneumonic plague. The abilities to disseminate
C Biomed Central

() 2011 Haiko et al; licensee BioMed Central Ltd. This is an Open Access article distributed under the terms of the Creative Commons Attribution License (http://creativecommons.org/licenses/by/2.0), which permits unrestricted use, distribution, and reproduction in any medium, provided the original work is properly cited. 
in the host and to cause high bacteremia are central for the transmission of the bacterium by the flea vector which feeds on contaminated blood. Y. pestis has been responsible for three human pandemics, which are estimated to have resulted in deaths of ca. 200 million humans [5]. As a bacterial species $Y$. pestis is young, and recent population genetic studies have shown that the bacterium diverged from its ancestral species, the gastrointestinal pathogen Yersinia pseudotuberculosis, only ca. 13000 years ago [6,7]. The genome of $Y$. pestis has evolved through gene decay, recombination, single nucleotide changes, genome rearrangements, and horizontal gene transfer by acquisition of two $Y$. pestis-specific plasmids, of which the plasmid pPCP1 (pPst/pPla) potentiates bacterial dissemination from the primary intradermal infection site into lymph nodes $[8,9]$. The decisive virulence factor encoded by PPCP1 is the surface protease plasminogen activator Pla. Deletion of pla attenuates $Y$. pestis millionfold in subcutaneously infected mice, whereas no difference is seen in intravenously infected mice [8]. Pla is specifically needed for establishment of bubonic plague $[10,11]$, and a critical role of Pla has been described in pneumonic plague where it enables localized growth of $Y$. pestis in the lungs [12].

Pla belongs to an outer membrane protease family of omptins that have been detected in several Gram-negative bacteria of different phylogenetic groups; these bacteria commonly infect animals or plants [13]. The omptin genes have spread through horizontal gene transfer by different mechanisms, and at least 16 members are known to date [13-16]. As omptin sequences are over $50 \%$ identical, they very likely fold similarly to the two structurally resolved members of the family, OmpT of $E$. coli [17] and Pla of $Y$. pestis [18]. Both OmpT and Pla form a 70- $\AA$ long $\beta$-barrel of elliptical cross-section with ten antiparallel transmembrane $\beta$-strands, five surfaceexposed loops (L1-L5) and four short periplasmic turns (T1-T4). The catalytic residues and the acidic, substratebinding pocket, as well as the lipopolysaccharide-binding site are conserved in omptins $[13,17,19]$. This is in accordance with the finding that the cleavage site preferences of omptin proteolysis, analyzed mostly with short peptide substrates, are very similar: omptins cleave after basic residues, preferably between two basic amino acids [20-23]. With larger substrates, however, omptins are functionally diverse, and their genes have undergone adaptive evolution to support the life style of the bacterial host $[14,15,24]$. Insertions and deletions that give slight variation in the molecular sizes of omptins are mostly located in the solvent-accessible surface structures L1-L5, and the differential substrate selectivities of individual omptin proteases appear to be dictated by these surface loops, as observed by loop swapping and omptin chimeras $[16,19,25,26]$.
During infection, Pla seems to increase virulence mainly by interfering with the human plasminogen/fibrinolytic system that is critical in cell migration across tissue barriers. Pla cleaves the circulating, abundant zymogen plasminogen by a single cut to active plasmin [8] and also degrades two natural inhibitors of the plasminogen system, the serpins $\alpha_{2}$-antiplasmin $\left(\alpha_{2} \mathrm{AP}\right)$ and plasminogen activator inhibitor 1 (PAI-1) $[16,19]$. Altogether, these activities result in uncontrolled plasmin activity. Plasmin is a broad-substrate protease involved in a number of (patho)physiological processes, of which fibrinolysis and damage of extracellular matrices contribute to bacterial dissemination within the host $[12,27,28]$. Pla has also non-proteolytic functions: it mediates invasion of $Y$. pestis into human epithelial and endothelial cells by an unknown mechanism as well as into mouse monocytes by binding to CD205 [29-31]. The Epo omptin is a close homolog of Pla, exhibiting $77 \%$ sequence identity, and it is encoded on a plasmid of the plant pathogen Erwinia pyrifoliae that causes tissue-destructive infection in pear trees [32]. The functions of Epo in plant pathogenesis have not been systematically studied, but serpinolytic activity in vitro was recently shown [16].

The claim of directed evolution and adaptive molecular evolution is that natural selection generates particularly evolvable enzymes in response to rapidly fluctuating selective conditions and that proteins that require fewest mutations to adapt to novel conditions are the most likely to survive environmental changes [33]. Evolvability has been argued to be a function of robustness, i.e., the capacity of a protein to withstand sequence variations without disruption of its binding or catalytic properties [33,34]. Mutational robustness is dependent on a protein's intramolecular interactions, and the omptin molecule fulfils the criteria of an evolvable protein as defined by O'Loughlin et al. [33]; the $\beta$ barrel is a sturdy membrane-embedded molecule with flexible surface loops that can tolerate deletions and insertions without compromising molecular stability $[35,36]$. Manifestations of the robustness of omptins include their unhurt catalytic activity after autocatalytic cleavage at L4 and the proteolytic specificity of omptin loop chimeras, which together have indicated that the five loops offer a flexible scaffold for recognition of different substrates $[16,19,25,26]$.

Evolutional studies on bacterial virulence factors highlight the importance of environment as an immense reservoir of evolvable potential progenitors of virulence factors [2]. Pla of $Y$. pestis and Epo of E. pyrifoliae belong to the same subfamily of omptins which also contains the omptins PgtE of Salmonella enterica, Kop of Klebsiella pneumoniae, and the omptin of Enterobacter $[15,16]$. These bacterial species infect mammals or plants or both, 
and have encountered mutual horizontal gene transfer. In this study we used the existing knowledge of omptin structure and function as a starting point for substitutional analysis, and addressed two specific questions on molecular adaptation: how many substitutions are needed and at which locations in Epo to gain a proteolytic function (plasminogen activation) and a non-proteolytic function (invasiveness) expressed by Pla.

\section{Results}

Substrate selectivity of Pla and Epo towards virulenceassociated human proteins

We began this study by comparing the activity of Pla and Epo in Pla-mediated functions that i) have been documented to be important in plague pathogenesis in vivo, such as plasminogen activation by cleavage, or ii) directly or indirectly enhance the virulence potential in vitro, such as degradation of $\alpha_{2} \mathrm{AP}$ and PAI- 1 and invasion to the human endothelial-like cell line. Mature Pla sequence differs from Epo by 65 amino acids, of which 31 are in protein regions located outward the outer membrane lipid bilayer. Of these 31 differences, 18 are located in the surface loops L1-L5 (Figure 1). For these assays, we expressed pla and epo in a well-defined laboratory host strain E. coli XL1 to avoid background problems with other virulence characteristics of $Y$. pestis and $E$. pyrifoliae, which either have been shown to or might interfere with the proteolytic activity of Pla or Epo [37-39].

A clear functional difference between Pla and Epo was observed in plasminogen activation: Pla activated

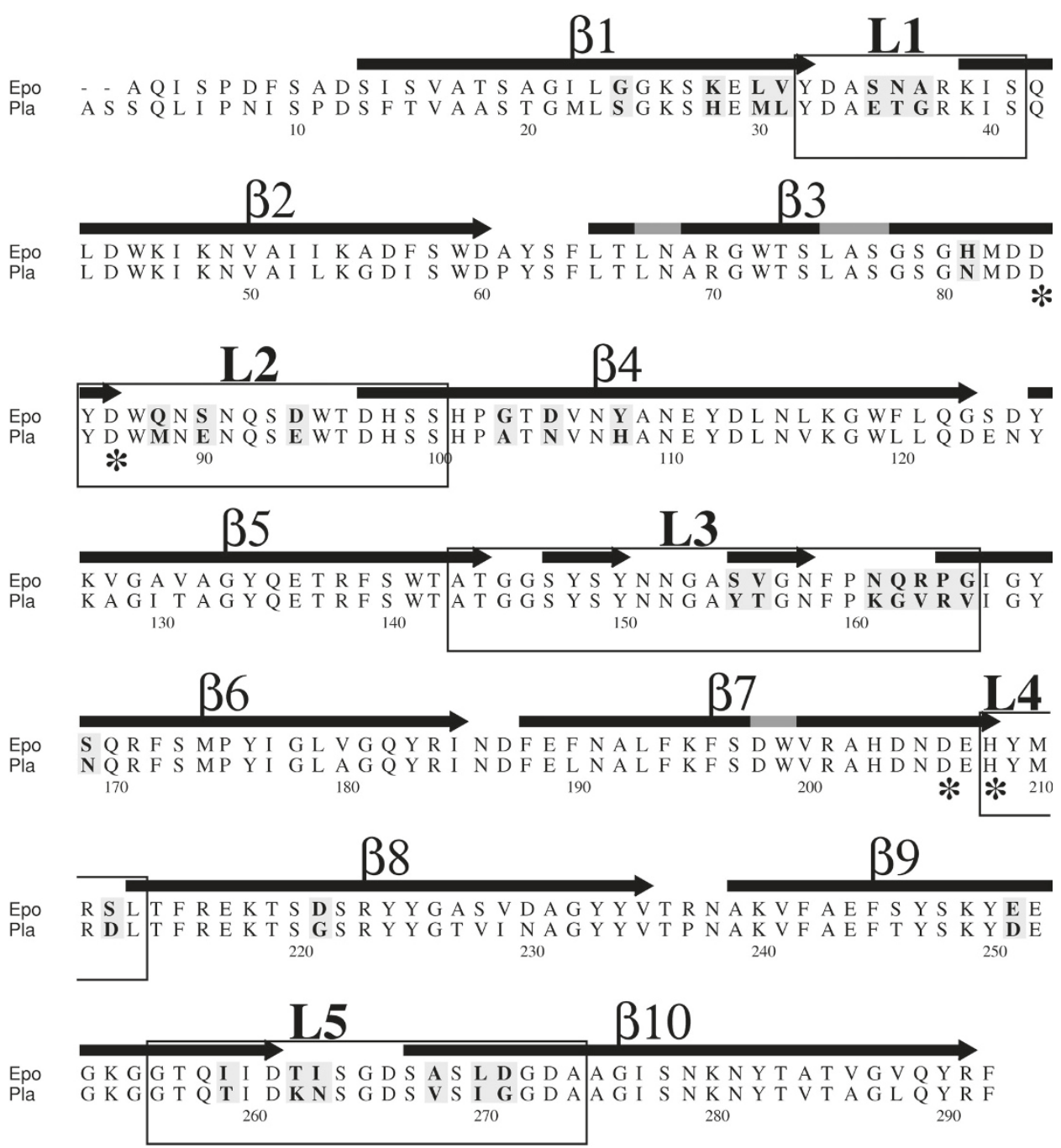

Figure 1 Sequence alignment of Epo and Pla. The $\beta$-strands and the extracellular loops are numbered, and the 31 differing amino acids in the extracellular side of the protein are shown in bold face in gray background. The catalytic residues are indicated with asterisks. The sequences were aligned with ClustalW [50], and the image was rendered with Alscript [51]. The secondary structures are defined by Stride [52], and they are based on the known Pla structure [18]. 
plasminogen efficiently but Epo very poorly during a 3-h cumulative measurement (Figure 2A). We next analyzed plasminogen degradation by Pla and Epo using Western blotting with two different antibodies: i) polyclonal antihuman plasminogen antibody that mainly detects the charged kringle domains in the heavy chain of plasmin, and ii) monoclonal anti-human plasminogen antibody that recognizes the catalytic domain in the light chain of plasmin (Figure 2B). Epo expressed by E. coli XL1 (pMRK4) degraded plasminogen but significantly more slowly than did Pla expressed in the same host strain in a 2-h incubation with plasminogen (Figure 2B). Further, Epo did not form detectable light chain of plasmin in a 3-h incubation with plasminogen, which however was

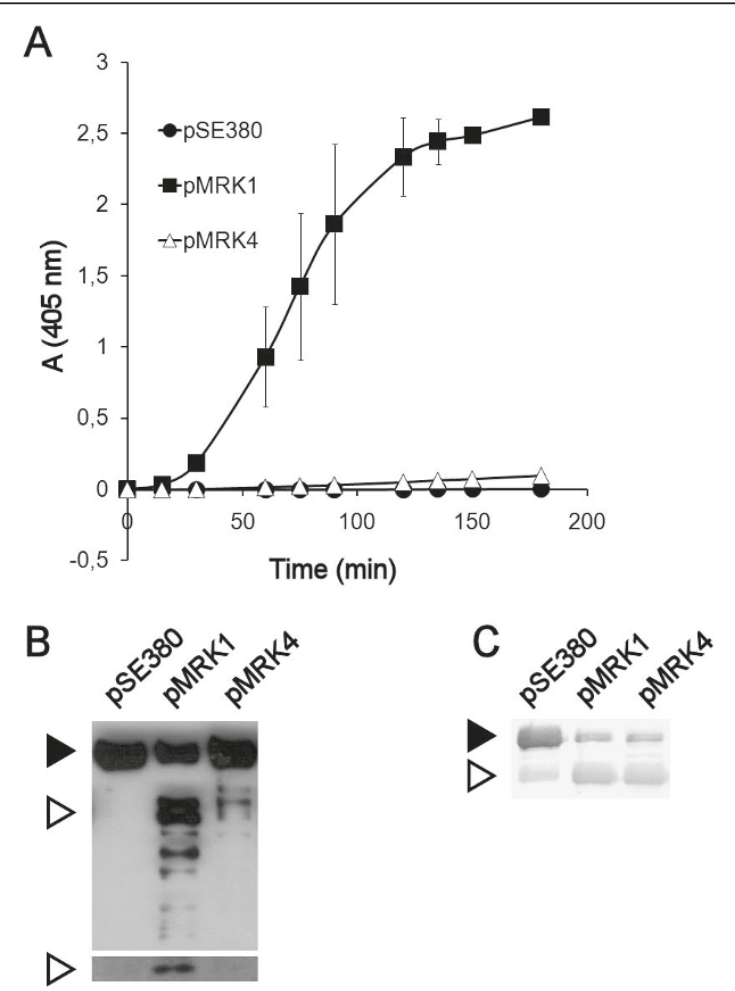

Figure 2 Comparison of Pla and Epo. Pla and Epo were compared in (A) plasminogen activation, (B) degradation of plasminogen, and (C) degradation of $\alpha_{2}$-antiplasmin. pla and epo were expressed in recombinant $E$. coli XL1. The strains are indicated with their plasmid names (see Table 1). In (A), plasmin formation was measured using a chromogenic plasmin substrate. The data are average of two independent assays with duplicate samples, and standard deviations are shown. In (B), Western blotting with antiplasminogen (top panel) and anti-plasminogen catalytic domain (bottom panel) antibodies is shown. The black arrowhead indicates plasminogen and the white arrowheads indicate cleaved plasminogen, the heavy chain (top panel) and the light chain (bottom panel) of plasmin. In (C), degradation of $\alpha_{2} A P$ was visualized with Western blotting using $\alpha_{2} A P$ antibody, and the black arrowhead indicates uncleaved $\alpha_{2} \mathrm{AP}$ and the white arrowhead indicates the degradation product. discernible with the Pla-expressing bacteria (Figure 2B). We have recently reported that Epo and Pla degrade the serpin PAI-1 [16], and recombinant E. coli with Pla or Epo also cleaved another serpin, $\alpha_{2}$ AP (Figure 2C). Thus, both omptins exhibited similar serpinolytic activity but differed in plasmin formation.

\section{Construction of Pla-Epo hybrid proteins}

In order to define the structural differences between Pla and Epo that govern the Pla-like plasminogen activation, we analyzed the sequence differences of Pla and Epo, especially at the surface accessible regions which are important for polypeptide substrate selectivity of omptins (see Figure 1) $[19,25]$. We generated Pla-Epo hybrid proteins by swapping amino acid residues in the loop regions differing between Pla and Epo sequences, and then expressed the proteins in E. coli XL1. We initially selected the residues to be substituted on the basis of their clustering and location at the loops or on the extracellular side on the protein. Our approach was to create cumulative substitutions, first at those loop regions where the most striking amino acid differences between Pla and Epo are, and then at the amino acids located on the extracellular side of the protein above the lipid bilayer. Initially, the substitutions and analyses were made in two directions: positive alteration from Epo towards Pla (i.e., gain of function) and negative alteration from Pla towards Epo (i.e., loss of function). The nomenclature of the hybrid-expressing plasmids is as follows: the first number tells the origin ( 1 for Pla, 4 for Epo), the next two numbers tell the number of substitutions made in the mature protein, and the possible change of $\beta$ strands is then given (see Table 1). E.g., pMRK431 denotes the Epo-based hybrid with 31 amino acid substitutions towards Pla and pMRK431 $\beta 2$ a hybrid additionally substituted for the $\beta 2$ strand. The hybrid proteins were expressed from the same high-copy plasmid in the same host strain; however, we also analyzed their expression by Western blotting of whole cells and of cell wall preparations using a cocktail of anti-Pla, anti-Epo, and anti-Pla loop-specific sera (Figure 3). These assays did not reveal substantial differences in the protein expression levels that would explain, according to our previous experience $[19,37,39]$, the observed differences in plasminogen activation. The assay however is semi-quantitative as the different hybrid proteins vary in reactivity with the antibodies.

\section{Plasminogen activation by Pla-Epo hybrid proteins}

Cumulative plasminogen activation assays [19], where the recombinant $E$. coli were incubated with plasminogen and the chromogenic plasmin substrate, were performed with Pla-Epo chimeras. The plasmid pMRK405 encodes the substitution of the loop 3 sequence 
Table 1 Bacterial strains and plasmids used in this study

\begin{tabular}{|c|c|c|}
\hline $\begin{array}{l}\text { Bacterial strain or plasmid } \\
\text { construct }\end{array}$ & Description & Reference \\
\hline Escherichia coli XL1 Blue MRF' & 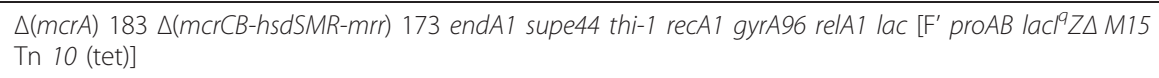 & Stratagene \\
\hline pSE380 & Expression vector, trc promoter, lacO operator, lacl, bla & Invitrogen \\
\hline pMRK1 & pla in pSE380 & [19] \\
\hline pMRK4 & epo in pSE380 & [16] \\
\hline pMRK105 & Pla with 5 aa from Epo: ${ }^{161}$ KGVRV $\rightarrow$ NQRPG & This study \\
\hline pMRK405 & Epo with 5 aa from Pla: ${ }^{159} \mathrm{NQRPG} \rightarrow$ KGVRV & This study \\
\hline pMRK110 & Pla with 10 aa from Epo: ${ }^{161} \mathrm{KGVRV} \rightarrow \mathrm{NQRPG},{ }^{262} \mathrm{KN} \rightarrow \mathrm{TI},{ }^{268} \mathrm{VSIG} \rightarrow \mathrm{ASLD}$ & This study \\
\hline pMRK410 & Epo with 10 aa from Pla: ${ }^{159} \mathrm{NQRPG} \rightarrow \mathrm{KGVRV},{ }^{260} \mathrm{TI} \rightarrow \mathbf{K N},{ }^{266} \mathbf{A S L D} \rightarrow$ VSIG & This study \\
\hline pMRK117 & $\begin{array}{l}\text { Pla with } 17 \text { aa from Epo: }{ }^{35} \mathrm{ETG} \rightarrow \mathrm{SNA},{ }^{88} \mathrm{MNE} \rightarrow \mathrm{QNS},{ }^{155} \mathrm{YT} \rightarrow \mathrm{SV}, \\
{ }^{268} \mathrm{VSIG} \rightarrow \mathrm{ASLD}\end{array}$ & This study \\
\hline pMRK417 & $\begin{array}{l}\text { Epo with } 17 \text { aa from Pla: }{ }^{33} \mathrm{SNA} \rightarrow \mathrm{ETG},{ }^{86} \mathrm{QNS} \rightarrow \mathrm{MNE},{ }^{153} \mathrm{SV} \rightarrow \mathrm{YT},{ }^{159} \mathrm{NQRPG} \rightarrow \mathrm{KGVRV},{ }^{260} \mathrm{TI} \rightarrow \mathrm{KN} \text {, } \\
{ }^{266} \rightarrow \mathrm{VSIG}\end{array}$ & This study \\
\hline pMRK431 & $\begin{array}{l}\text { Epo with } 31 \text { aa from Pla: }{ }^{33} \mathrm{SNA} \rightarrow \mathrm{ETG}^{86} \mathrm{QNS} \rightarrow \mathrm{MNE},{ }^{153} \mathrm{SV} \rightarrow \mathrm{YT},{ }^{159} \mathrm{NQRPG} \rightarrow \mathrm{KGVRV},{ }^{260} \mathrm{Tl} \rightarrow \mathrm{KN}, \\
{ }^{260} \mathrm{ASLD} \rightarrow \mathrm{VSIG},{ }^{26} \mathrm{KELV} \rightarrow \mathrm{HEML},{ }^{101} \mathrm{GTDVNY} \rightarrow \text { ATNVNH, I257T, H79N, D92E, S210D, S167N, } \\
\text { D219G, E249D, G22S }\end{array}$ & This study \\
\hline pMRK431 $\beta 1$ & $\begin{array}{l}\text { Epo with } 42 \text { aa from Pla + the signal sequence: N-terminus from Pla until aa 45; the rest similar to } \\
\text { pMRK } 431\end{array}$ & This study \\
\hline PMRK4 $4 \beta 1 \mathrm{~L} 1$ & Epo with the $\mathrm{N}$-terminus from Pla until aa 45 & This study \\
\hline PMRK410ß1L1 & pMRK410 with the $\mathrm{N}$-terminus from Pla until aa 45 & This study \\
\hline pMRK417ß 1L1 & pMRK417 with the N-terminus from Pla until aa 45 & This study \\
\hline pMRK431ß2 & pMRK431 with $\beta 2$ from Pla & This study \\
\hline pMRK431ß4 & pMRK431 with $\beta 4$ from Pla & This study \\
\hline pMRK431ß5 & pMRK431 with $\beta 5$ from Pla & This study \\
\hline pMRK431 $\beta 8+9$ & pMRK431 with $\beta 8 \& \beta 9$ from $\mathrm{Pla}$ & This study \\
\hline pMRK431 $\beta 10$ & pMRK431 with $\beta 10$ from Pla & This study \\
\hline
\end{tabular}

Bold text indicates the novel substitutions compared to previous hybrid. aa, amino acid.

${ }^{159} \mathrm{NQRPG}$ of Epo to the sequence ${ }^{161}$ KGVRV of Pla (see Figure 1 and Table 1), and this substitution in Epo resulted in efficient plasminogen activation (Figure 4A). The reverse substitution in pla, encoded in plasmid pMRK105, nearly completely abolished plasminogen activation by recombinant $E$. coli (Figure 4B). We next analyzed the effect of the further substitutions ${ }^{260} \mathrm{TI} / \mathrm{KN}$ and ${ }^{266}$ ASLD/VSIG which are located at L5 in Epo and encoded in pMRK410, and this hybrid protein was nearly as effective as Pla in plasminogen activation (Figure 4A). The corresponding substitutions in Pla encoded in plasmid pMRK110 gave a protein with very low plasminogen activation (Figure 4B). Further loop substitutions in Epo-based plasmid pMRK417, i.e.

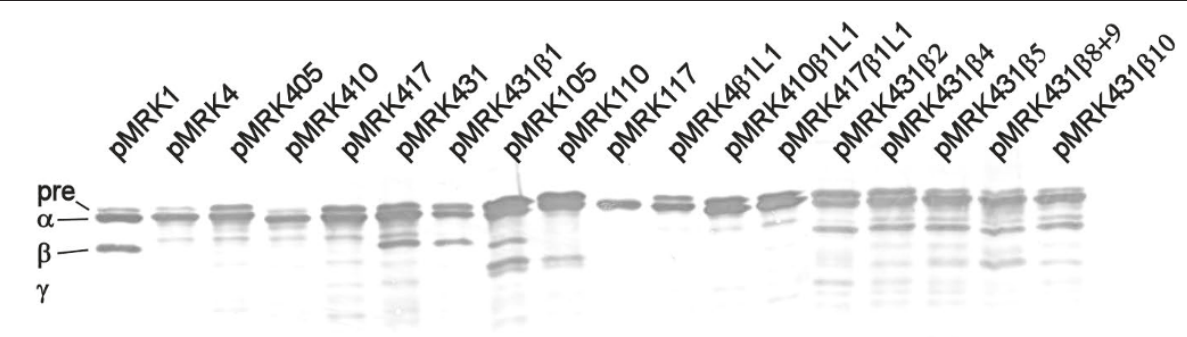

Figure 3 Analysis of expression of Pla-Epo hybrid proteins in E. coli. Expression of the Pla-Epo-hybrid proteins was analyzed by Western blotting of whole cells with a mixture of anti-Pla, anti-Epo, and anti-Pla-loop antisera. The constructs are indicated with their plasmid names (see Table 1). Migration distance of the Pla isoforms are indicated; pre-Pla is the immature form of Pla, $\alpha$-Pla and $\gamma$-Pla are mature Pla proteins with differing conformations, and $\beta$-Pla is the mature autocleaved form of Pla. 

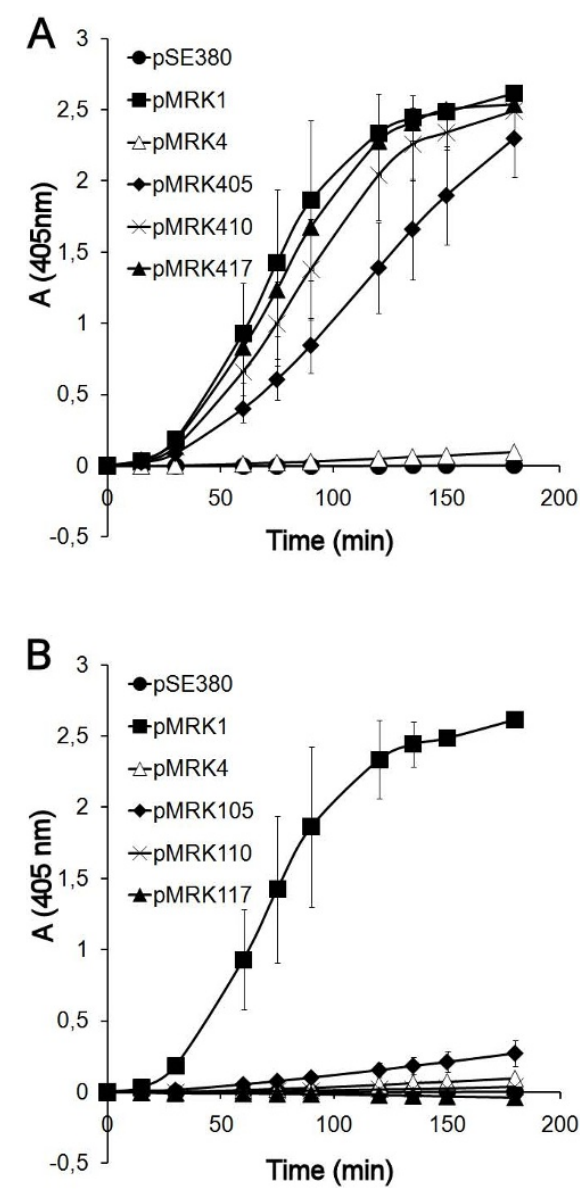

Figure 4 Cumulative plasminogen activation by Pla-Epo hybrid proteins expressed in E. coli. (A) Pla-Epo hybrids with cumulative substitutions towards Pla. (B) Pla-Epo hybrids with cumulative substitutions towards Epo. Inserts show the plasmid constructs (see Table 1). The data are average from two independent assays with duplicate samples, and standard deviations are shown.

${ }^{33} \mathrm{SNA} / \mathrm{ETG}$ at L1, ${ }^{86} \mathrm{QNS} / \mathrm{MNE}$ at L2 and ${ }^{153} \mathrm{SV} / \mathrm{YT}$ at L3, only slightly improved plasminogen activation. The reverse substitutions in pMRK117 gave a hybrid protein that did not activate plasminogen at all. Thus, the substitution of 10 out of the 290 amino acids in the mature Epo protein, located at L3 and L5, was enough to significantly alter its protease substrate selectivity towards activation of human plasminogen.

The stepwise accumulation of substitutions onto the Epo molecule was continued onwards from pMRK417 to pMRK431, where all 31 residues that differ between Epo and Pla and that are located on the extracellular side of the outer membrane on the protein have been substituted towards Pla. These substitutions in Epo did not change the cumulative plasminogen activation or the fragmentation pattern of plasminogen (data not shown). We recently reported that the single substitution I259T at loop 5 differentiates Pla of the pandemic
$Y$. pestis lineages (which have PlaT259) from the Pla isoform of the ancestral lineages (PlaI259). This substitution decreases Pla-mediated cleavage of plasmin light chain that contains the catalytic domain, and thus increases the stability of the formed plasmin [26]. An increase in plasmin stability and formation of the plasmin light chain after the I259T substitution was also observed in the series of Pla-Epo hybrid proteins in the present study.

\section{Structural analysis}

To gain physical insight into the observed functional differences in proteolysis, we studied the roles of the swapped residues in the crystal structure of Pla [18] and the homology model of Epo. The immediate environment of the residues substituted in pMRKX10, where $\mathrm{X}$ is either 1 or 4, is shown in Table 2 and Figure 5. The first five substituted amino acids occur at the very end of the long loop L3 and the following five are found at the tip of L5 and in the early, solvent-accessible part of strand $\beta 10$. There are no direct contacts between ${ }^{159} \mathrm{NQRPG} /$ KGVRV and ${ }^{260} \mathrm{TI} / \mathrm{KN}$ or ${ }^{266}$ ASLD/VSIG, but both the $\mathrm{L} 3$ and the $\mathrm{L} 5$ residues interact with the intervening $\mathrm{L} 4$. The $\beta 7$-strand before $\mathrm{L} 4$ contains active site residues D206 and H208, and is fully conserved between Epo and Pla except for one residue (S210/D212; see Figure 1).

The five residues of the first substitution site are the longest consecutive difference between Pla and Epo. In the Pla structure, V163 at L3 packs together with apolar residues P160 at L3 and M210 at L4 at the inside of the barrel, while the L3 residues N159, Q160, and R161 in Epo point towards the water phase (Figure 5). The distinct functional effect of pMRK405 substitutions could

Table 2 Molecular environment of the substituted sites in Pla structure and Epo model

\begin{tabular}{|c|c|c|c|c|}
\hline & Pla & Contact & Epo & Contact \\
\hline \multirow[t]{5}{*}{ MRKX05 } & K161 & solvent & N159 & Q160, R161 \\
\hline & G162 & - & Q160 & N159, R161 \\
\hline & V163 & $\mathrm{p} 160, \underline{\mathrm{m} 210}$ & R161 & N159, Q160, p158 \\
\hline & R164 & $\underline{\mathrm{y} 209}, \mathrm{t} 142$ & P162 & R161 \\
\hline & V165 & H101, a143, g145, i166 & G163 & - \\
\hline \multirow[t]{6}{*}{ MRKX10 } & $K 262$ & $\underline{\mathrm{e} 207}, \mathrm{t} 214$ & $T 260$ & $\underline{\mathrm{S} 210}, \underline{\mathrm{t} 212,} \mathbf{d} 259$ \\
\hline & $N 263$ & $\underline{\mathrm{D} 212}, \mathrm{~d} 261$ & 1261 & $d 259$ \\
\hline & V268 & 1270 & $A 266$ & i257 \\
\hline & $s 269$ & $q 258$ & $s 267$ & $q 256$ \\
\hline & 1270 & $t 257, v 268, a 274$ & $L 268$ & - \\
\hline & $G 271$ & - & D269 & a32, g270 \\
\hline
\end{tabular}

Residues whose side chains come within $4 \AA$ of the central amino acid are listed. The amino acids in each loop are indicated in different font styles, L3 (G145-V165) in bold, L4 (H208-L213) underlined and L5 (G255-A274) in italics in Pla numbering.

Residues that are conserved at the site are shown in lower case letters, and the non-conserved ones in capital letters. 


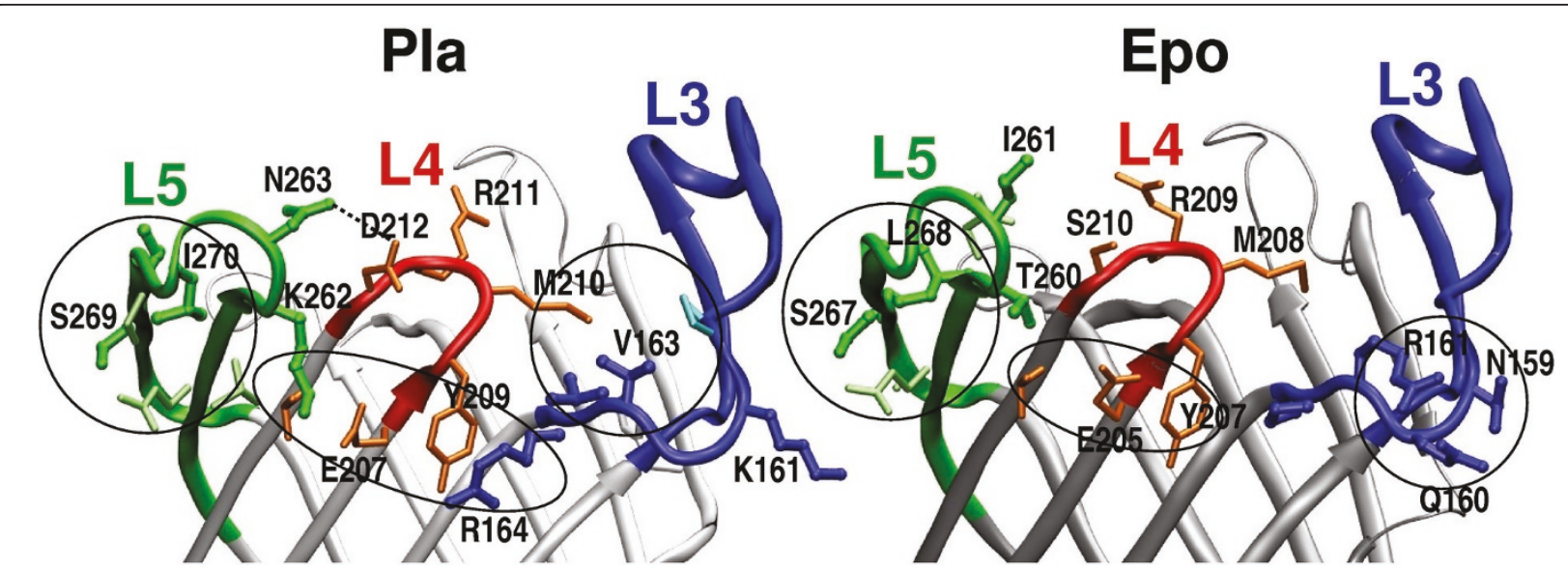

Figure 5 Analysis of the ten substituted residues in Pla structure (left) and Epo model (right). $L 3$ and its residues are colored blue, $L 4$ red and L5 green. The substituted residues are drawn thick and in a darker shade, and the other discussed residues thin and in lighter shade. The hydrogen bond between D212 and N263 in Pla is marked with a dashed line, and three interaction areas circled in both structures. Counting from left: a tight cluster of hydrophobic residues in L5; a polar quintet between $L 5$, $L 4$ and $L 3$ in Pla and a $L 4$ triplet in Epo; hydrophobic contacts within the barrel opening in Pla and polar residues at the outside of the barrel in Epo.

well reflect changes in size of the binding pocket and differences in the electrostatic properties of the substrate docking surface.

The incremental substitutions of pMRK410 occur in L5. K262 and N263 are located in the very tip of L5, and in the Pla structure, both residues are in contact with $\mathrm{L} 4$. Most intriguingly, N263 forms a hydrogen bond with Pla-specific D212 in L4. Such an interaction is not possible between the corresponding residues in Epo, S210 and I261. Moreover, K262 of Pla points towards E207 in $\beta 7$. This glutamate, and the threonine and the tyrosine hydrogen-bonded to it are identical in Pla and Epo, but the polar triplet at the outside of the barrel gets extended and strengthened in Pla by K262 (L5) from one side and by R164 (L3) from the other (Figure 5). The corresponding Epo-residues T260 and P162 are decisively smaller, and do not reach to the L4 residues. pMRK410 construct also includes swapping of ${ }^{268}$ VSIG/ ${ }^{266}$ ASLD in L5. These residues lie in a small $\beta$-structure [18], supposedly in the aqueous phase above the lipopolysaccharide layer. The residues in both proteins form similar interactions with their neighbors in same loop, except the Epo-specific D269 that in the model hydrogen bonds to a backbone carbonyl in the $\beta 1$-strand. The end of loop L5 and the beginning of L1 are rather irregular; both areas differ between Pla and Epo, and a side chain-backbone hydrogen bond would stabilize the loops relative to each other. In the Pla structure, L1 residues D33-R38 form a network of hydrogen bonds.

\section{Serpinolytic activity of Pla-Epo hybrids}

To study the effect of structural changes on degradation of another substrate, we next analyzed the degradation of PAI- 1 and $\alpha_{2}$ AP by the Pla-Epo chimeras. Pla and Epo degraded PAI- 1 and $\alpha_{2}$ AP in a similar manner (Figure 6). Recombinant E. coli strains (pMRK405), (pMRK410), and (pMRK417) cleaved PAI-1 and $\alpha_{2} \mathrm{AP}$ more efficiently than did E. coli with Pla or Epo (Figure 6). On the other hand, the hybrids with further substitutions, encoded in plasmids pMRK431 and pMRK431 $\beta 1$, caused slower degradation of both PAI-1 and $\alpha_{2}$ AP, similar to that seen with Pla and Epo. The Pla-derived hybrid proteins, encoded by plasmids pMRK105, pMRK110, and pMRK117, were severely decreased in their serpinolytic activity compared to both Pla and Epo.

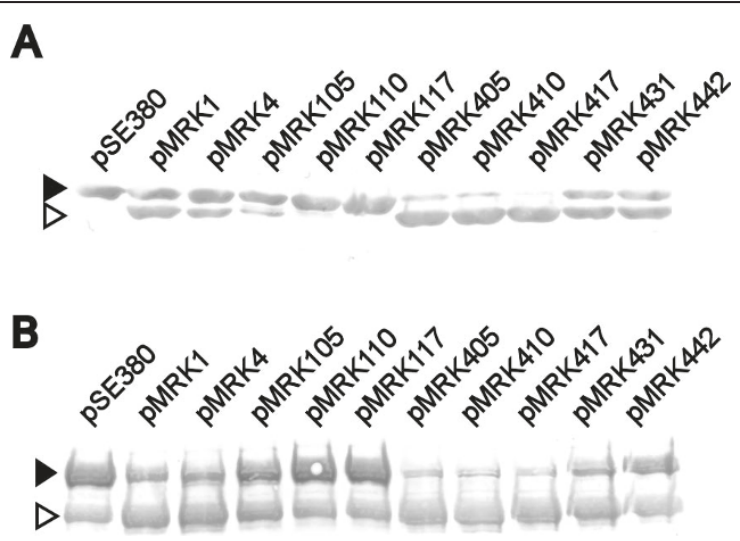

Figure 6 Degradation of serpins by $E$. coli expressing the PlaEpo hybrid proteins. Degradation of PAl-1 (A) and $\alpha_{2} A P(B)$ in a 2$\mathrm{h}$ incubation was analyzed by Western blotting. The black arrowhead indicates uncleaved serpin and the white arrowhead indicates the degradation product. The strains are indicated with their plasmid names (see Table 1). 
Thus, serpinolytic activity and plasminogen activation seem to require different amino acids in the omptins.

\section{Turning Epo into an invasin}

Invasiveness of $Y$. pestis into human epithelial and endothelial cells is a putative virulence-associated property of Pla $[29,30]$, which was detected in a gentamicin protection assay with $E$. coli (pMRK1) expressing Pla but not with E. coli (pMRK4) expressing Epo (Table 3). To compare gain of invasiveness to gain of plasminogen activation, we tested the Pla-Epo chimeras for their effect on invasion into the ECV304 cell line. E. coli expressing chimeras up to pMRK417 gave a modest increase in bacterial invasiveness. Surprisingly, E. coli XL1 (pMRK431) also showed only a low but clearly positive level of invasiveness, indicating that substitution of all extramembranous residues differing between Pla and Epo, sufficient to gain Plalike plasminogen activation, was not enough to turn Epo into an efficient invasin. Strikingly, further substitutions in the transmembrane region of Epo in pMRK431ß1 (expressing all 31 Pla-specific residues located above the membrane and the remaining five residues, F14, T15, A18, T20, and $\mathrm{M} 22$, from the $\mathrm{N}$-terminal $\beta 1$-strand and the irregular region before the $\beta 1$-strand) induced invasiveness to the level seen with $E$. coli (pMRK1) expressing Pla. The hybrid encoded in pMRK431ß1 was similar to pMRK431 in enhancing plasminogen activation by E. coli (data not shown). Fusion of the same 5' pla DNA (thus including the $\beta 1$-strand and the L1) into epo or the hybrids pMRK410 or pMRK417 did not induce similar invasiveness (Table 3), indicating that the Pla-like omptin surface

Table 3 Results of the invasion assay

\begin{tabular}{|c|c|c|c|}
\hline E. coli XL1 & $\begin{array}{l}\text { Average invasion } \\
\text { percentage }\end{array}$ & $\begin{array}{l}\text { Number of } \\
\text { assays }\end{array}$ & Range \\
\hline pSE380 & 0,0 & 33 & $0-0.3$ \\
\hline pMRK1 & $4,4^{* *}$ & 33 & $0.7-24.8$ \\
\hline pMRK4 & 0,0 & 9 & $0-0.05$ \\
\hline pMRK417 & $0,1^{*}$ & 9 & $0-0.2$ \\
\hline pMRK431 & 0,5 & 6 & $0-1.9$ \\
\hline pMRK431 $\beta 1$ & $5,4^{* *}$ & 14 & $0.4-16.3$ \\
\hline pMRK4 $\beta 1 \mathrm{~L} 1$ & 0,0 & 4 & $0-0.04$ \\
\hline pMRK410 $\beta 1 \mathrm{~L} 1$ & 0,1 & 6 & $0.02-0.3$ \\
\hline pMRK417ß1L1 & 1,2 & 6 & $0.04-5.4$ \\
\hline pMRK431ß2 & 0,4 & 5 & $0.1-0.8$ \\
\hline pMRK431 $\beta 4$ & 0,4 & 5 & $0.02-1.2$ \\
\hline pMRK431ß5 & 0,3 & 5 & $0.03-0.9$ \\
\hline pMRK $431 \beta 8+9$ & 0,1 & 5 & $0.02-0.2$ \\
\hline pMRK431 310 & $0,2^{*}$ & 6 & $0.06-0.3$ \\
\hline
\end{tabular}

Gentamicin protection assay with the human ECV304 cell line. The results are given as the percentage of the intracellular vs. the total added bacteria. The strains have been compared to E. coli XL1 (pSE380) to calculate significant differences with t-scores. ${ }^{*} \mathrm{p}<0.05,{ }^{* *} \mathrm{p}<0.01$. is needed and the amino terminus alone does not potentiate invasion. Further, substitution of the other $\beta$-strands to pMRK431 construct did not influence invasiveness (Table 3). We substituted in pMRK431 the $\beta$ strands 2,4 , 5,8 and 9 together, and 10, that vary most in the $\beta$-strand sequences of Pla and Epo.

\section{Discussion}

Erwinia species and $Y$. pestis colonize different habitats and have a very different pathogenic potential for humans. Bacterial virulence is normally dependent on several factors, and presence of Pla, although it is an exceptionally multifunctional and powerful virulence factor [8,10-12,16,19,26-28,30,31] does not alone explain the devastating virulence of $Y$. pestis. Our study however gives an example of how a single, specific virulence factor of the plague bacterium might have structurally adapted to its present form. Our results do not give a direct roadmap to the Pla molecular structure but rather describe how easily the substrate specificity of an omptin can evolve and how functions that are pathogenic in a novel environment can be gained. The role of surface loops in Pla proteolysis has earlier been documented by loop swapping [16,26], and our results extend these findings by a more detailed substitutional analysis and by also conferring invasiveness onto a close ortholog of Pla.

Serpins are important regulators of cellular proteolysis and share structural features, which include a similar, exposed reactive center loop that binds to and inhibits the catalytic center of target proteases [40]. The omptins in the Pla subfamily all cleave specifically the reactive center loop of the PAI-1 [16]. Here we found that Epo can cleave the serpin $\alpha_{2} \mathrm{AP}$, which is a shared target of Pla and PgtE $[19,41]$. These findings indicate that serpinolytic activity is a common function in the Pla subfamily of omptins and might give selection advantage for survival and spread of bacteria in their hosts by increasing proteolysis in tissues. In contrast, omptins of the OmpT subfamily, OmpT of E. coli and SopA of Shigella, do not cleave PAI-1, despite high sequence similarity, conserved fold and active site structure [16]. It is interesting to note that the Pla-Epo hybrid proteins encoded in pMRK405, pMRK410, and pMRK417 were more efficient than Pla or Epo in PAI-1 degradation. Also, further substitution towards Pla, encoded in pMRK431 and pMRK431 $\beta 1$, led to hybrid proteins that exhibited an activity similar to that of Pla and Epo. Acquisition of novel substrate specificities by proteases often leads to broad-specificity variants by initially favoring mutations that increase the conformational flexibility and destabilize specialized active site structures. thus reducing specificity for the original targets [33]. The observation that intermediate Pla-Epo hybrid proteins were more 
efficient in PAI-1 degradation is in line with the assumption that serpinolytic activity is a property of a progenitor omptin in this subfamily and that Pla and Epo have diverged from this progenitor to have novel functions. The structural explanation for the increased efficiency is however unknown. The activating cleavage in plasminogen occurs between the R-V bond, while in PAI- 1 and probably also in $\alpha_{2}$ AP the cleavage occurs between R-M in the reactive center loop. Specialization to plasminogen cleavage by Pla seems to correlate with several stabilizing interactions between the surface loops, formed by Pla-specific residues.

A molecular explanation for the altered plasminogen activation encoded by pMRK410 remains speculative without more specific molecular data about omptintarget complexes, and more generally, because the in vivo mobility of the surface structures of the omptin barrel. Still, the structural analysis presented here suggests a possible explanation. Most importantly, we see multiple loop-loop interactions involving Pla-specific residues. These would result in reduced loop flexibility and a better defined binding pocket, promoting productive substrate binding. Second, we see a binding pocket altered in size and hydrophobicity. The pMRKX05 (where $\mathrm{X}$ is either 1 or 4 ) substitutions ${ }^{161} \mathrm{KGVRV} /$ NQRPG occur at one long end of the elliptical barrel opening, and the ${ }^{268}$ VSIG/ASLD at the other. This seems to imply that the polypeptide substrates bind across the long axis of the omptin pore, and the pMRKX10 mutations change the docking surface. Altogether, the Pla structure seems to allow close approach by the substrate, and the active site to be well stabilized by interloop interactions. Assuming this is the reason for the observed differences in plasminogen cleavage versus serpinolysis, it means that plasminogen binding and cleavage require a "preformed", tighter binding pocket.

The protein sites identified in this study enable the omptin to initiate activating cleavage of plasminogen but further substitutions are needed to stabilize plasmin. Swapping of loops between Pla and PgtE of S. enterica has revealed that $\mathrm{L} 5$ and the $\mathrm{N}$-terminal region including L1 are crucial for the gelatinase activity by PgtE [25]. The same regions and in addition L3 seem to be important in the activation of pro-matrix metalloproteinase 9 by PgtE [25]. A residue deserving special attention is T259 of Pla, which is situated in L5 and faces into the barrel opening next to F215. We recently described that a single substitution at this site, T259I that is typical for ancestral $Y$. pestis lineages, increases the cleavage of the light chain of plasmin, thus decreasing the stability of the formed plasmin [26]. The conservation of this mutation in pandemic isolates indicates that pla, after being acquired by $Y$. pestis, has evolved through a single nucleotide change to better support plasmin formation. The pPCP1 plasmid also encodes pesticin and pesticin immunity protein and is evolutionary related to colicin plasmids, in particular pColE1[42]. It is possible that the pPCP1 plasmid has evolved through fusion of a pla progenitor gene and a ColE1like plasmid.

The omptins autoprocess themselves at different protein sites [15]. Formation of $\beta$-Pla-like peptide by Epo hybrid proteins was detected by $E$. coli expressing pMRK431 that encodes the substitution of all 31 differences between Pla and Epo at their surface-exposed parts. This indicates that autoprocessing is dependent on surface loops. Unexpectedly, these substitutions only slightly improved the invasiveness of recombinant $E$. coli. This indicates that the proteolysis and the invasiveness involve different regions of the omptin molecule and that the invasiveness, which among omptins has been detected only with Pla [15], is a more complex function than the proteolytic specificity. The plasmid pMRK431 $\beta 1$, constructed from pMRK431 to also encode the amino terminal residues 1-45 containing the $\beta 1$-strand, induced similar level of invasiveness as seen with E. coli pMRK1. However, Pla amino terminus alone in Epo did not convert Epo to an invasin. At present we do not have an explanation for the requirement of transmembrane regions to induce invasion by Epo. Our hypothesis was that this might indicate TonB-dependency in invasion. TonB functions in energy coupling between the cytoplasmic and the outer membrane and is important in transport functions across the bacterial cell wall [43]. The Pla and Epo sequences contain a homolog to the TonB box [44] at the amino terminus $\left({ }^{11}\right.$ DSFTVAA in Pla and ${ }^{9}$ DSISVAT in Epo). A role for TonB in Pla-mediated invasion however seems unlikely, as expression of Pla or Epo in a tonB-deletion of E. coli XL1 mutant did not alter their invasiveness (T. Lehti and J. Haiko, unpublished). One possibility is that invasion requires membrane fusion which could differ between Epo and Pla, as outer membrane $\beta$-barrels are known to vary in their incorporation into different lipid micelles [45]. It is also possible that changes in membrane-embedded residues have subtle effects on the orientation and activity of surface residues that make contact with the target cell. Finally, as a molecular event invasion is complex and may require several steps (adhesion, membrane fusion, host cell responses) that involve more than one omptin protein region. This is in line with the finding that extensive substitution was required to induce invasiveness into Epo.

\section{Conclusions}

The omptin $\beta$-barrel fold has spread through horizontal gene transfer in Gram-negative bacteria, and their 
pathogenetic functions and potential vary significantly and correlate well with the pathogenetic mechanisms of the host bacterial species. This implies that the omptins have adapted and contribute to the life style of the bacteria, while they also have retained common functions such as serpinolytic activity. Pla and plasminogen activation are central in plague pathogenesis, and among the omptins, Pla is by far the most efficient plasminogen activator while other omptins either cleave plasminogen poorly or not at all $[15,19,26]$. Change of a few Epo amino acids to those in Pla conferred a plague virulence function on the omptin from a plant pathogen. The mature sequences of Pla and Epo differ at 65 out of $292 / 290$ residues, and we observed that the substitution ${ }^{159}$ NQRPG/KGVRV at L3 alone caused a substantial improvement in the plasminogen activation ability of Epo when expressed in E. coli. Further substitutions ${ }^{260} \mathrm{TI} / \mathrm{KN}$ and ${ }^{266} \mathrm{ASLD} / \mathrm{VSIG}$ at L5 improved the initial plasminogen activation close to the level seen with Plaexpressing E. coli. Structural analysis indicated that differing residues at L3 and L5 in Pla and Epo interact with L4 in different ways, leading to differences in the substrate binding pocket. Substitutions at L3 and L5 did not yet turn Epo into a Pla-like invasin, which required more extensive substitutions both at the surface-exposed part of the protein and the membrane-embedded $\mathrm{N}$ terminal region, the $\beta 1$-strand. Our findings are summarized in Figure 7.

\section{Methods}

\section{Bacterial strains and plasmids}

Bacterial strains and plasmids are listed in Table 1. pla and epo were available from previous work $[19,26]$. Derivatives of pla and epo were cloned by recombinant twostep PCR as described earlier [19,25] and expressed in the inducible pSE380 vector in E. coli XL1-Blue MRF as described earlier $[19,37]$. The nucleotide sequences were confirmed by sequencing. The recombinant $E$. coli strains were cultivated overnight at $37^{\circ} \mathrm{C}$ in Luria broth $(10 \mathrm{ml})$ supplemented with glucose $(0.2 \% \mathrm{wt} / \mathrm{vol})$, ampicillin $(100 \mu \mathrm{g} / \mathrm{ml})$, and tetracycline $(12.5 \mu \mathrm{g} / \mathrm{ml})$. For protein expression, the culture was pelleted, suspended in $150 \mu \mathrm{l}$ of phosphate- buffered saline (PBS, $\mathrm{pH} 7.1$ ), and plated on Luria plates containing $5 \mu \mathrm{M}$ isopropyl- $\beta$ D-thiogalactopyranoside (IPTG) and antibiotics as described above. For the assays, bacteria were collected in PBS, pelleted, and adjusted to an optical density at $600 \mathrm{~nm}$ of 1.2 (corresponding to ca. $10^{9}$ cells $/ \mathrm{ml}$ ) or 2.0 $\left(2 \times 10^{9}\right.$ cells $\left./ \mathrm{ml}\right)$.

\section{Plasminogen activation}

Human Glu-plasminogen (4 $\mu \mathrm{g}$; American Diagnostica), was mixed with $8 \times 10^{7}$ bacteria in PBS in a total volume of $174 \mu \mathrm{l}$ in microtiter plate (Nunc)

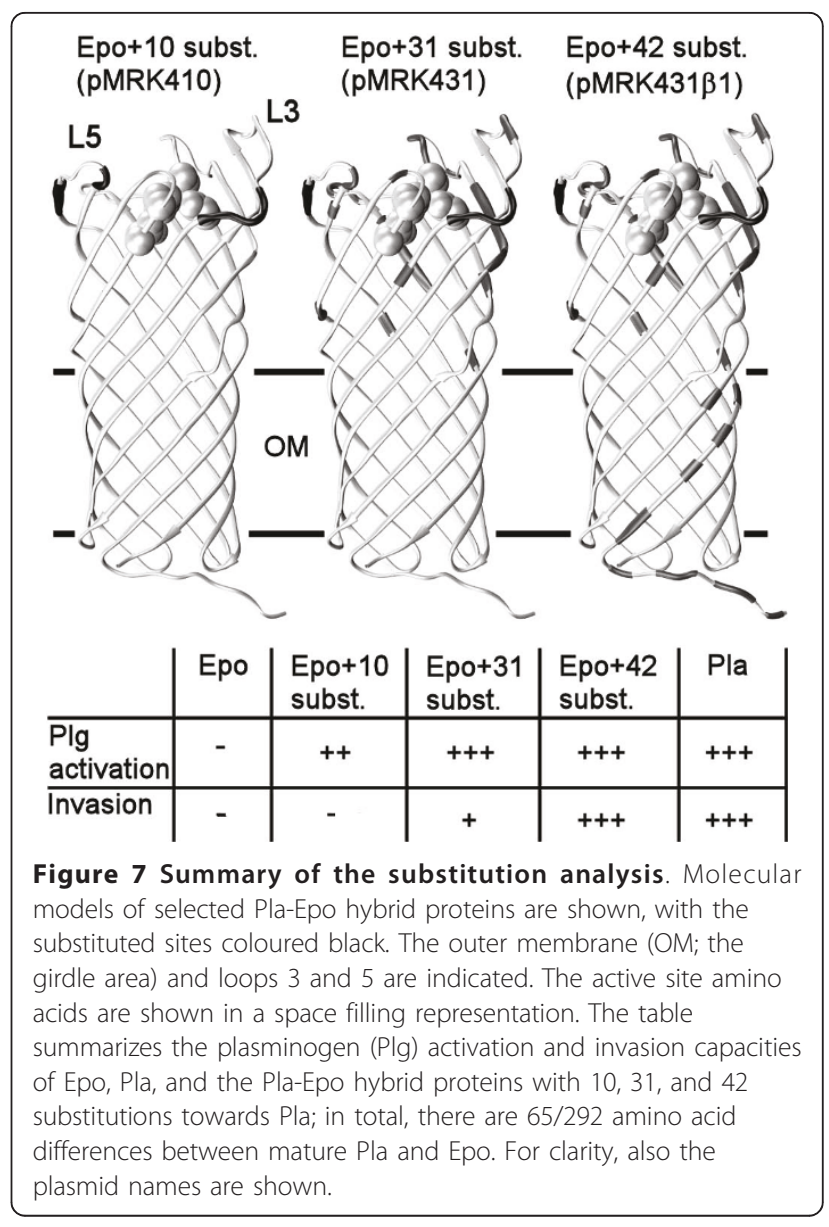

wells, and $30 \mu \mathrm{l}$ of the chromogenic plasmin substrate Val-Leu-Lys-p-nitroaniline dihydrochloride (S-2251, $2.5 \mathrm{mg} / \mathrm{ml}$; Chromogenix) was added. Plasmin formation at $37^{\circ} \mathrm{C}$ was measured in a microtiter plate reader at $405 \mathrm{~nm}$.

\section{Degradation of $\alpha_{2}$-antiplasmin and plasminogen}

Cleavage of human $\alpha_{2}$ AP ( $0.8 \mu \mathrm{g}$; Calbiochem) was tested as detailed earlier $[19,41]$, by using $3 \times 10^{7}$ bacteria and the incubation for $2 \mathrm{~h}$ at $37^{\circ} \mathrm{C}$. Plasminogen degradation with anti-human plasminogen antibody (American Diagnostica) was assessed by incubating 2.5 $\mu \mathrm{g}$ human Glu-plasminogen and $4 \times 10^{7}$ bacteria in PBS for $2 \mathrm{~h}$ shaking at $37^{\circ} \mathrm{C}$. The cells were pelleted, and the supernatants were boiled with SDS-PAGE loading buffer. Samples $(10 \mu \mathrm{l})$ were run in a $12 \%$ (wt/vol) SDSPAGE gel and transferred onto a polyvinylidene difluoride membrane (GE Healthcare). The detection was done with polyclonal anti-human plasminogen antibody (1 $\mathrm{mg} / \mathrm{ml}$, diluted 1:1000), peroxidase-conjugated antirabbit IgG (1:2500; GE Healthcare), and enhanced chemiluminescence detection reagents (GE Healthcare) according to the manufacturer's instructions. The 
membrane was exposed to X-ray film (Agfa) for $15 \mathrm{~s}$. In assays where plasminogen cleavage was detected with monoclonal anti-human plasminogen catalytic domain antibody (R\&D Systems), the following modifications were made: Glu-plasminogen $(3 \mu \mathrm{g})$ and $4.8 \times 10^{7}$ bacteria were incubated for $3 \mathrm{~h}$. After incubation, supernatant samples of $15 \mu \mathrm{l}$ were electrophoresed and detection was done with the monoclonal antibody $(500 \mu \mathrm{g} / \mathrm{ml}$, diluted 1:500) and peroxidase-conjugated anti-mouse IgG (GE Healthcare; 1:1000). The exposure time was $5 \mathrm{~min}$.

\section{Degradation of PAl-1/vitronectin complex}

Recombinant active human PAI-1 (2.5 $\mu \mathrm{g}$; American Diagnostica) and human plasma Vn (5 $\mu$; Promega) were incubated for $1 \mathrm{~h}$ at $37^{\circ} \mathrm{C}$ in a volume of $5 \mu \mathrm{l}$ to form a complex [46]. Bacteria $\left(20 \mu \mathrm{l}, 4 \times 10^{7}\right.$ cells) in PBS were incubated with the complex for $2 \mathrm{~h}$ at $37^{\circ} \mathrm{C}$. The bacteria were pelleted, and the supernatants were run in a $12 \%$ (wt/vol) SDS-PAGE gel, and PAI-1 and $\mathrm{Vn}$ were detected by Western blotting with polyclonal anti-PAI-1 (1:5000, Calbiochem) or anti-Vn (1:1000, Calbiochem) antibodies, alkaline-phosphataseconjugated anti-rabbit immunoglobulin G (Dako) and phosphatase substrate.

\section{Detection of Pla-Epo hybrids with anti-Pla, anti-Epo, and anti-Pla loop sera}

The cell envelope samples were made by sonicating bacteria $\left(10^{9}\right.$ cells $\left./ \mathrm{ml}\right)$ in PBS on ice with $2.5 \mathrm{mM}$ ethylene diamine tetra-acetic acid (EDTA). The remaining cells were pelleted, and the supernatants were centrifuged further. The cell envelopes in the supernatants were pelleted and suspended in PBS and SDS-PAGE loading buffer. Whole-cell samples were made by boiling the bacteria $\left(10^{9}\right.$ cells $\left./ \mathrm{ml}\right)$ with SDS-PAGE loading buffer, and the samples were loaded into a $12 \%$ (wt/vol) SDSPAGE gel, electrophoresed, and transferred onto a nitrocellulose membrane. The proteins were detected with a mixture of anti-His 6 -Pla antiserum, antipeptide sera against Pla loops 3 and 5 [19], and anti-His ${ }_{6}$-Epo antiserum (1:1000). The detection was done with alkalinephosphatase-conjugated anti-rabbit immunoglobulin G (IgG; Dako) and phosphatase substrate.

\section{Invasion into ECV304 cells}

Endothelial-like ECV304 cell line was cultivated in Medium 199 (Gibco) supplemented with 10\% fetal calf serum (Gibco) and $2 \mathrm{mM}$ L-glutamine (Gibco) and grown at $37^{\circ} \mathrm{C}$ with $5 \% \mathrm{CO}_{2}$ for 3-4 days. Bacterial invasion into ECV304 cells was studied using gentamicin protection assay [47]. ECV304 cells were grown at 24well plate (Nunc) for 3-4 days. The eukaryotic cells were washed with PBS and Medium 199 was added. Bacteria $\left(10^{5}\right.$ cells) in Medium 199 were added, and the plate was centrifuged $(128 \times \mathrm{g}, 10 \mathrm{~min})$. The cells were incubated at $37^{\circ} \mathrm{C}$ with $5 \% \mathrm{CO}_{2}$ for $2 \mathrm{~h}$. The wells were washed with $\mathrm{PBS}$, and the extracellular bacteria were killed with gentamicin $(100 \mu \mathrm{g} / \mathrm{ml})$. ECV304 cells were lysed with $0.2 \%$ Triton X-100, and the number of intracellular bacteria was determined by viable counting. The invasion percentages were calculated by comparing the amount of the bacteria incubated with the eukaryotic cells to the amount of intracellular bacteria.

\section{Molecular modelling of protein structures}

The omptin structures were modelled on the basis of the crystal structures of Pla [18] (PDB code $2 \times 4 \mathrm{~m}$ ) with program Modeller 9v7 [48] and analyzed visually with program VMD [49].

\section{Acknowledgements}

We thank Raili Lameranta and Janne Tynell for technical assistance and Enni Korhonen and Jaakko Lyytinen for help in statistical analysis. This work was financially supported by the European Union Network of Excellence EuroPathoGenomics program (grant number CEE LSHB-CT-2005-512061), Research Foundation of the University of Helsinki, Viikki Graduate School in Molecular Biosciences, Magnus Ehrnrooth Foundation, and the Academy of Finland (grant numbers 116507 and 130202).

\section{Author details}

'Division of General Microbiology, Department of Biosciences, P.O. Box 56, FI 00014 University of Helsinki, Finland. ${ }^{2}$ Neuroscience Center, P.O. Box 56, FI 00014 University of Helsinki, Finland.

\section{Authors' contributions}

$J H$ performed the laboratory experiments. LL constructed the homology model and analyzed the structures. BWW helped with the design of the study and writing. TKK supervised the work and mainly wrote the manuscript. All authors participated in planning the work and writing the manuscript and read and approved the final manuscript.

Received: 7 October 2010 Accepted: 11 February 2011

Published: 11 February 2011

\section{References}

1. Pallen MJ, Nelson KE, Preston GM: Bacterial pathogenomics. Washington, DC, USA: ASM Press; 2007

2. Seifert HS, Dirita VJ: Evolution of microbial pathogens. Washington, DC, USA: ASM Press; 2006.

3. Hacker J, Kaper JB: Pathogenicity islands and the evolution of microbes. Annual Review of Microbiology 2000, 54:641-679.

4. Yen YT, Kostakioti M, Henderson IR, Stathopoulos C: Common themes and variations in serine protease autotransporters. Trends Microbiol 2008, 16(8):370-379.

5. Perry RD, Fetherston JD: Yersinia pestis - etiologic agent of plague. Clin Microbiol Rev 1997, 10(1):35-66.

6. Achtman M, Aurth K, Morelli G, Torrea G, Guiyoule A, Carniel E: Yersinia pestis, the cause of plague, is a recently emerged clone of Yersinia pseudotuberculosis. Proc Natl Acad Sci USA 1999, 96(24):14043-14048.

7. Achtman M, Morelli G, Zhu P, Wirth T, Diehl I, Kusecek B, Vogler AJ, Wagner DM, Allender CJ, Easterday WR, Chenal-Francisque V, Worsham P, Thomson NR, Parkhill J, Lindler LE, Carniel E, Keim P: Microevolution and history of the plague bacillus, Yersinia pestis. Proc Natl Acad Sci USA 2004, 101(51):17837-17842.

8. Sodeinde OA, Subrahmanyam YV, Stark K, Quan T, Bao Y, Goguen JD: A surface protease and the invasive character of plague. Science 1992, 258(5084):1004-1007. 
9. Wren BW: The yersiniae-a model genus to study the rapid evolution of bacterial pathogens. Nat Rev Microbiol 2003, 1(1):55-64.

10. Sebbane F, Jarrett CO, Gardner D, Long D, Hinnebusch BJ: Role of the Yersinia pestis plasminogen activator in the incidence of distinct septicemic and bubonic forms of flea-borne plague. Proc Natl Acad Sci USA 2006, 103(14):5526-5530

11. Sebbane F, Lemaitre N, Sturdevant DE, Rebeil R, Virtaneva K, Porcella SF, Hinnebusch BJ: Adaptive response of Yersinia pestis to extracellular effectors of innate immunity during bubonic plague. Proc Natl Acad Sci USA 2006, 103(31):11766-11771.

12. Lathem WW, Price PA, Miller VL, Goldman WE: A plasminogen-activating protease specifically controls the development of primary pneumonic plague. Science 2007, 315(5811):509-513.

13. Rawlings ND, Morton FR, Kok CY, Kong J, Barrett AJ: MEROPS: the peptidase database. Nucleic Acids Research 2008, 36:D320-D325.

14. Hritonenko V, Stathopoulos C: Omptin proteins: an expanding family of outer membrane proteases in Gram-negative Enterobacteriaceae. Mol Membr Biol 2007, 24(5-6):395-406

15. Haiko J, Suomalainen M, Ojala T, Lähteenmäki K, Korhonen TK: Breaking barriers - attack on innate immune defences by omptin surface proteases of enterobacterial pathogens. Innate Immunity 2009, 15(2):67-80.

16. Haiko J, Laakkonen $L$, Juuti $K$, Kalkkinen N, Korhonen TK: The omptins of Yersinia pestis and Salmonella enterica cleave the reactive center loop of plasminogen activator inhibitor 1. J Bacteriol 2010, 192:4553-4561.

17. Vandeputte-Rutten L, Kramer RA, Kroon J, Dekker N, Egmond MR, Gros P: Crystal structure of the outer membrane protease OmpT from Escherichia coli suggests a novel catalytic site. EMBO J 2001 20(18):5033-5039.

18. Eren E, Murphy M, Goguen J, van den Berg B: An active site water network in the plasminogen activator Pla from Yersinia pestis. Structure 2010, 18(7):809-818.

19. Kukkonen $M$, Lähteenmäki K, Suomalainen M, Kalkkinen N, Emödy L, Lång $\mathrm{H}$, Korhonen TK: Protein regions important for plasminogen activation and inactivation of alpha2-antiplasmin in the surface protease Pla of Yersinia pestis. Mol Microbiol 2001, 40(5):1097-1111.

20. Dekker N, Cox RC, Kramer RA, Egmond MR: Substrate specificity of the integral membrane protease OmpT determined by spatially addressed peptide libraries. Biochemistry 2001, 40(6):1694-1701

21. McCarter JD, Stephens D, Shoemaker K, Rosenberg S, Kirsch JF, Georgiou G: Substrate specificity of the Escherichia coli outer membrane protease OmpT. J Bacteriol 2004, 186(17):5919-5925.

22. Hwang BY, Varadarajan N, Li H, Rodriguez S, Iverson BL, Georgiou G Substrate specificity of the Escherichia coli outer membrane protease OmpP. J Bacteriol 2007, 189(2):522-530.

23. Agarkov A, Chauhan S, Lory PJ, Gilbertson SR, Motin VL: Substrate specificity and screening of the integral membrane protease Pla. Bioorg Med Chem Lett 2008, 18(1):427-431.

24. Kukkonen $\mathrm{M}$, Korhonen TK: The omptin family of enterobacterial surface proteases/adhesins: from housekeeping in Escherichia coli to systemic spread of Yersinia pestis. Int J Med Microbiol 2004, 294(1):7-14.

25. Ramu P, Lobo LA, Kukkonen M, Bjur E, Suomalainen M, Raukola H Miettinen M, Julkunen I, Holst O, Rhen M, Korhonen TK, Lähteenmäki K: Activation of pro-matrix metalloproteinase-9 and degradation of gelatin by the surface protease PgtE of Salmonella enterica serovar Typhimurium. Int J Med Microbiol 2008, 298(3-4):263-278.

26. Haiko J, Kukkonen M, Ravantti JJ, Westerlund-Wikström B, Korhonen TK: The single substitution 1259T, conserved in the plasminogen activator Pla of pandemic Yersinia pestis branches, enhances fibrinolytic activity. J Bacteriol 2009, 191(15):4758-4766.

27. Lähteenmäki K, Virkola R, Saren A, Emödy L, Korhonen TK: Expression of plasminogen activator pla of Yersinia pestis enhances bacterial attachment to the mammalian extracellular matrix. Infect Immun 1998, 66(12):5755-5762.

28. Degen JL, Bugge $T H$, Goguen JD: Fibrin and fibrinolysis in infection and host defense. J Thromb Haemost 2007, 5(Suppl 1):24-31.

29. Cowan C, Jones HA, Kaya YH, Perry RD, Straley SC: Invasion of epithelial cells by Yersinia pestis: evidence for a Y. pestis-specific invasin. Infect Immun 2000, 68(8):4523-4530.
30. Lähteenmäki K, Kukkonen M, Korhonen TK: The Pla surface protease/ adhesin of Yersinia pestis mediates bacterial invasion into human endothelial cells. FEBS Lett 2001, 504(1-2):69-72.

31. Zhang SS, Chae GP, Zhang P, Bartra SS, Plano GV, Klena JD, Skurnik M, Hinnebusch BJ, Chen T: Plasminogen activator Pla of Yersinia pestis utilizes murine DEC-205 (CD205) as a receptor to promote dissemination. J Biol Chem 2008, 283(46):31511-31521.

32. McGhee GC, Schnabel EL, Maxson-Stein K, Jones B, Stromberg VK, Lacy GH, Jones AL: Relatedness of chromosomal and plasmid DNAs of Erwinia pyrifoliae and Erwinia amylovora. Appl Environ Microbiol 2002, 68(12):6182-6192.

33. O'Loughlin TL, Patrick WM, Matsumura I: Natural history as a predictor of protein evolvability. Protein Eng Des Sel 2006, 19(10):439-442.

34. Wagner A: Robustness, evolvability, and neutrality. FEBS Lett 2005, 579(8):1772-1778.

35. Koebnik R, Locher KP, Van Gelder P: Structure and function of bacterial outer membrane proteins: barrels in a nutshell. Mol Microbiol 2000, 37(2):239-253

36. Schulz GE: $\beta$-barrel membrane proteins. Curr Opin Struct Biol 2000, 10(4):443-447.

37. Kukkonen $M$, Suomalainen $M$, Kyllönen $P$, Lähteenmäki K, Lång H, Virkola R, Helander IM, Holst O, Korhonen TK: Lack of O-antigen is essential for plasminogen activation by Yersinia pestis and Salmonella enterica. Mol Microbiol 2004, 51(1):215-225.

38. Galván EM, Lasaro MA, Schifferli DM: Capsular antigen fraction 1 and Pla modulate the susceptibility of Yersinia pestis to pulmonary antimicrobial peptides such as cathelicidin. Infect Immun 2008, 76(4):1456-1464.

39. Suomalainen $M$, Lobo LA, Brandenburg $K$, Lindner B, Virkola R, Knirel YA, Anisimov AP, Holst O, Korhonen TK: Temperature-induced changes in the lipopolysaccharide of Yersinia pestis affect plasminogen activation by the Pla surface protease. Infect Immun 2010, 78(6):2644-2652.

40. Gils A, Declerck PJ: Structure-function relationships in serpins: current concepts and controversies. Thromb Haemost 1998, 80(4):531-541.

41. Lähteenmäki K, Kyllönen P, Partanen L, Korhonen TK: Antiprotease inactivation by Salmonella enterica released from infected macrophages. Cell Microbiol 2005, 7(4):529-538.

42. Pilsl H, Killmann H, Hantke K, Braun V: Periplasmic location of the pesticin immunity protein suggests inactivation of pesticin in the periplasm. J Bacteriol 1996, 178(8):2431-2435.

43. Postle K, Kadner RJ: Touch and go: Tying TonB to transport. Mol Microbiol 2003, 49(4):869-882.

44. Postle K, Larsen RA: TonB-dependent energy transduction between outer and cytoplasmic membranes. Biometals 2007, 20(3-4):453-465.

45. Burgess NK, Dao TP, Stanley AM, Fleming KG: Beta-barrel proteins that reside in the Escherichia coli outer membrane in vivo demonstrate varied folding behavior in vitro. J Biol Chem 2008, 283(39):26748-26758.

46. Wiman B, Almquist A, Sigurdardottir O, Lindahl T: Plasminogen activator inhibitor 1 (PAI) is bound to vitronectin in plasma. FEBS Lett 1988, 242(1):125-128.

47. Tang P, Foubister V, Pucciarelli MG, Finlay BB: Methods to study bacterial invasion. J Microbiol Methods 1993, 18(3):227-240.

48. Sali A, Blundell TL: Comparative protein modelling by satisfaction of spatial restraints. J Mol Biol 1993, 234(3):779-815.

49. Humphrey W, Dalke A, Schulten K: VMD - Visual Molecular Dynamics. J Molec Graphics 1996, 14:33-38.

50. Larkin MAG, Blackshields G, Brown NP, Chenna R, McGettigan PA, McWilliam H, Valentin F, Wallace IM, Wilm A, Lopez R, Thompson JD, Gibson TJ, Higgins DG: Clustal W and Clustal X version 2.0. Bioinformatics 2007, 23:2947-2948.

51. Barton GJ: ALSCRIPT: a tool to format multiple sequence alignments. Protein Engineering 1993, 6(1):37-40.

52. Frishman D, Argos P: Knowledge-based protein secondary structure assignment. Proteins 1995, 23(4):566-579.

doi:10.1186/1471-2148-11-43

Cite this article as: Haiko et al:: Molecular adaptation of a plantbacterium outer membrane protease towards plague virulence factor Pla. BMC Evolutionary Biology 2011 11:43. 